\title{
Ovarialkarzinom
}

\section{Aktuelle und zukünftige Behandlungsoptionen}

\begin{abstract}
Auf dem Update 2010 Ovarialkarzinom stellte Andreas du Bois, Essen, die aktuellen Leitlinien zur Primärtherapie des Ovarialkarzinoms vor. In frühen Stadien (FIGO I-IIA) komme der operativen Entfernung des Tumors die höchste Bedeutung zu, wichtig für den Therapierfolg sei dabei ein optimales chirurgisches Staging. Zur Verbesserung des rezidivfreien und des Gesamtüberlebens sei außerdem, außer bei Stadium IA G1, eine adjuvante Chemotherapie angezeigt - platinhaltig und sechs Zyklen umfassend.
\end{abstract}

Auch in fortgeschrittenen Krankheitsstadien (FIGO IIB-IV) ist der postoperative Tumorrest sowohl ein ausschlaggebender als auch ein beeinflussbarer Prognosefaktor. „Entscheidend für eine möglichst lange Überlebenszeit einer Patientin mit fortgeschrittenem Ovarialkarzinom ist die Kombination von State-of-theArt Operation und State-of-theArt Chemotherapie“, so du Bois. Standardregime sei auch hier eine Kombination aus Carboplatin und Paclitaxel über sechs Zyklen.
Der Stellenwert der Rezidivchirurgie lasse sich nicht durch prospektive Studiendaten mit hohem Evidenzniveau belegen, erklärte Jalid Sehouli, Berlin. Retrospektive Daten sprächen aber dafür, dass die komplette Tumorresektion auch beim Rezidiv der wichtigste Prognosefaktor sei. Sehouli betonte jedoch, dass eine Operation nicht eine systemische Therapie ersetzen könne. Bei refraktären Rezidiven sei eine Monochemotherapie z.B mit Topotecan (Hycamtin ${ }^{\circ}$ ) am effektivsten, bei platinsensitiven Rezidiven zeige die Kombination Carboplatin/ Doxorubicin die beste Wirkung.

Neben laufenden Studien zur Anwendbarkeit verschiedener Angiogenese- und Tyrosinkinaseinhibitoren beim Ovarialkarzinom, erwähnte Jacobus Pfisterer, Solingen, die Möglichkeit des Einsatzes von PARP (Poly-ADPRibose-Polymerase)-Inhibitoren wie Olaparib. Diese können Mutationsstatus-abhängig die DNA-Reparatur in Tumorzellen gezielt verhindern, und die Zellen dadurch auch sensitiver für andere Therapien machen. Derzeit wird in einer Phase-II-Studie die Wirksamkeit der zusätzlichen Gabe von Olaparib zu einer Chemotherapie überprüft. $\quad$ skf

Symposium am 30. Oktober 2010 in Berlin; Veranstalter: GlaxoSmithKline

\section{Metastasiertes Mammakarzinom AGO erneuert Empfehlungen zur Capecitabin-Therapie}

\begin{abstract}
Die Arbeitsgemeinschaft für Gynäkologische Onkologie (AGO) erneuert in ihren aktuellen Leitlinien die Empfehlungen für die Monotherapie mit Capecitabin (Xeloda ${ }^{\oplus}$ ) in der Behandlung des metastasierten Mammakarzinoms[ www.ago-online.org].
\end{abstract}

Die effiziente antitumorale Wirksamkeit der CapecitabinMonotherapie in der therapeutisch schwierigen Situation nach Anthrazyklin- und/oder Taxan-Versagen ist gut belegt. So erzielte Capecitabin in der Standarddosierung $\left(2.500 \mathrm{mg} / \mathrm{m}^{2} / \mathrm{Tag}\right)$ in einer multizentrischen Phase-IIStudie bei massiv vorbehandelten Patientinnen im metastasierten Krankheitsstadium eine objektive Ansprechrate von 20\%. Das mediane Gesamtüberleben lag bei 12,8 Monaten bei einem medianen progressionsfreien Überleben von drei Monaten [Blum JL et al.
J Clin Oncol 1999;17:485-93]. So empfiehlt die AGO auch 2011 sowohl nach Anthrazyklin- als auch nach Taxan- und AnthrazyklinVorbehandlung Capecitabin mit höchstem Empfehlungsgrad (++).

Auch in der First-Line-Therapie des metastasierten Mammakarzinoms sind die Ergebnisse der intermittierenden Capecitabin-Monotherapie gut: In einer offenen, multizentrischen und prospektiven Phase-II-Studie MoniCa erhielten 161 Patientinnen Capecitabin in reduzierter Dosierung $\left(2.000 \mathrm{mg} / \mathrm{m}^{2} / \mathrm{Tag}\right)$ bis zum Fortschreiten der Erkrankung oder dem Auftreten inakzeptabler Toxizitäten: Das progressionsfreie Überleben betrug median 7,9 und das Gesamtüberleben median 18,6 Monate. Zudem bestätigt die Studie erneut die gute Verträglichkeit der Capecitabin-Monotherapie: $87 \%$ der behandelten Patientinnen vertrugen die Therapie ohne Dosismodifikation [Kaufmann $\mathrm{M}$ et al. Eur J Cancer 2010;46:3184-91]. Angesichts dieser Datenlage hatte die AGO in 2010 erstmals eine positive Bewertung (+) von Capecitabin mono in der palliativen FirstLine-Therapie des metastasierten Mammakarzinoms ausgesprochen. Auch diese Empfehlung wurde in den aktuellen AGO-Leitlinien erneut bestätigt. $\mathrm{db}$ 